\title{
Imaging of angiogenesis: from morphology to molecules and from bench to bedside
}

\author{
Ambros J. Beer • Xiaoyuan Chen
}

Published online: 17 July 2010

(C) Springer-Verlag 2010

\begin{abstract}
Angiogenesis is one of the hallmarks of cancer and is increasingly in the focus of oncological research. Judah Folkman first articulated the importance of angiogenesis for tumor growth in 1971 [1]. He stated that the growth of solid tumors remains restricted to $2-3 \mathrm{~mm}$ in diameter until the onset of angiogenesis. Although this hypothesis first was strongly criticized, angiogenesis soon became one of the most important fields of research in oncology. Up to now, subsequent investigations have identified more than 20 angiogenic growth factors, their receptors and signal transduction pathways. Moreover, endogenous angiogenesis inhibitors have been discovered, and the cellular and molecular characterization of the angiogenic phenotype in human cancers has been achieved [2,3]. Although the results of the first clinical trials using angiogenesis inhibitors in oncology were disappointing, encouraging results have been achieved in the past few years with the vascular endothelial growth factor (VEGF) antibody Avas$\operatorname{tin}^{\circledR}$ in combination with standard cytotoxic chemotherapy. This combined antiangiogenic-cytotoxic therapy has been proven to be successful first in metastasized colorectal cancer, and subsequently also in breast cancer and nonsmall cell lung cancer [4-7]. This success of targeted antiangiogenic therapy will spur the demand for imaging
\end{abstract}

\footnotetext{
A. J. Beer $(\bowtie)$

Department of Nuclear Medicine, Klinikum rechts der Isar, Technische Universität München,

Munich, Germany

e-mail: ambros.beer@tum.de

X. Chen $(\varangle)$

Laboratory of Molecular Imaging and Nanomedicine,

National Institute of Biomedical Imaging and Bioengineering,

National Institutes of Health,

Bethesda, MD, USA

e-mail: Shawn.Chen@nih.gov
}

modalities for assessment of the angiogenic cascade, which is the topic of this supplement "Imaging of Angiogenesis." Imaging of angiogenic activity might on the one hand help in the process of developing novel antiangiogenic drugs in the preclinical setting. Moreover, in the clinical setting, imaging of angiogenesis might be useful for assessment of the optimum dose of new antiangiogenic agents and for early response evaluation. Up to now, clinical trials with conventional cytotoxic chemotherapeutic agents have mainly used morphological imaging to provide indices of therapeutic response, mostly computed tomography (CT) or magnetic resonance imaging (MRI). Bidimensional measurements of the maximum tumor extension are mainly used to estimate changes in response to the investigational therapy as compared with a baseline measure. Through standardization of these measurements by introducing the RECIST criteria in the year 2000, considerable progress has been achieved [8]. However, as antiangiogenic agents lead to a stop of tumor progression rather than to tumor shrinkage, the approach of measuring tumor response by a reduction of tumor size is not applicable and might take months or years to become apparent. Therefore, there is great interest in identifying reliable biomarkers of early tumor response to noncytotoxic drugs [9]. Imaging techniques could potentially be used as such a biomarker and could provide an early indicator of effectiveness at a functional or molecular level. Concerning morphological imaging of the vasculature, conventional X-ray angiography and digital subtraction angiography (DSA) are widely used in the clinical arena; however, they do not allow for assessment of the microvasculature and thus are not commonly used for response evaluation of antiangiogenic therapies. More promising is the assessment of changes in hemodynamic parameters such as blood flow, blood volume, or vessel 
permeability. They may be effective biomarkers for response evaluation, because antiangiogenic therapies are designed to affect the abnormal blood vessels found in tumors. Current clinical trials employ various imaging techniques for this purpose, mostly dynamic contrast-enhanced MRI (DCE MRI), and less often ultrasound, PET (especially with $\left[{ }^{15} \mathrm{O}\right]$ water), and dynamic contrast-enhanced CT (DCE CT) [10]. In the future, targeting specific molecular markers of angiogenesis might also be used for response assessment of antiangiogenic therapies like the VEGF pathway or cell surface markers like the integrin $\alpha v \beta 3$.

In this supplement of the European Journal of Nuclear Medicine and Molecular Imaging, the whole array of imaging techniques for assessment of angiogenesis on a morphological, functional, and molecular level are presented. In the first section of the supplement, Kiessling et al. [11] discuss the assessment of the structure of angiogenic vasculature, like with microCT and dedicated small animal ultrasound devices or even optoacoustic tomography.

In the next section, four articles deal with the different modalities for functional imaging of angiogenesis, which by now is still the most widely used imaging method for assessment of angiogenesis in the clinic. The manuscript by Laking and Price [12] reviews the most recent clinical results on PET and SPECT imaging of perfusion and also hypoxia. While in general they are technically more demanding than MRI or CT techniques, radiotracer techniques and especially PET with $\left[{ }^{15} \mathrm{O}\right] \mathrm{H}_{2} \mathrm{O}$ allow for quantitative assessment of blood flow and are often used as reference methods for other imaging techniques. In the next paper, Brix et al. [13] provide an overview on DCE MRI and $\mathrm{CT}$ techniques for assessment of perfusion in their manuscript, which are currently the most widely applied methods for imaging of angiogenesis in clinical trials. Another paper by Martirosian et al. [14] deals with MRI techniques providing perfusion-related contrast weighting without administration of contrast media. Especially arterial spin labeling (ASL) techniques are interesting in this respect and may provide a noninvasive tool for assessment of tissue perfusion rates in vivo. The section on functional imaging of angiogenesis is concluded by the manuscript by Cosgrove and Lassau [15] reporting on ultrasound techniques for imaging of perfusion.

The next section deals with imaging of molecular markers of tumor vasculature, which is a very promising approach in preclinical studies and might play an important role in the future clinical environment as well. Haubner et al. [16] report on the latest data concerning PET imaging of molecular markers of angiogenesis, such as integrin $\alpha \mathrm{v} \beta 3$ or VEGF. Dijkgraaf and Boerman [17] correspondingly review the advances in SPECT imaging of molecular targets of angiogenesis. The paper by Lanza et al. [18] explores the imaging options of using vascular-constrained contrast platform technologies, while Eisenblätter et al. [19] focus on optical imaging techniques for the assessment of angiogenesis. Eisenbrey and Forsberg [20] report on the role of ultrasound for molecular imaging of angiogenesis. The section is completed by the paper by Liu and Peng [21], which describes the use of inorganic nanomaterials for imaging of angiogenesis, including semiconductor quantum dots (QDs), single-walled carbon nanotubes (SWNTs), upconversion nanoparticles (UCNPs), and surfaceenhanced Raman scattering (SERS) nanoparticles.

The supplement is concluded by the paper by Zweifel and Padhani [22], which summarizes the currently available data on response assessment of antiangiogenic drugs with DCE MRI and gives a perspective on its future use.

At this point we would like to thank all the authors for their participation in this project and for their excellent work! With growing clinical use of targeted antiangiogenic therapies and the rising demand for noninvasive assessment of angiogenic activity, the time is right in our opinion for a comprehensive overview of the different aspects of "Imaging of Angiogenesis" presented in this supplement of the European Journal of Nuclear Medicine and Molecular Imaging.

In summary, a multitude of imaging techniques are available for the assessment of vasculature and angiogenesis on a structural, functional, and molecular level and are described in this supplement. All these methods have been successfully used preclinically and will hopefully aid in antiangiogenic drug development in animal studies. Up to now, only imaging of functional hemodynamic parameters (e.g., $\mathrm{K}^{\text {trans }}$ ), blood flow, and blood volume is currently used in the clinical arena to evaluate antiangiogenic and cytotoxic chemotherapies. Concerning imaging of molecular parameters of angiogenesis, only a few radiotracers have been used in humans up to now, and their role in assessment of antiangiogenic therapies is still unsettled. The integrin $\alpha \mathrm{v} \beta 3$ is still the most extensively used angiogenesis target for imaging, and ongoing clinical trials will hopefully show its potential in the near future. Concerning the optimum imaging technique for clinical use, the radiotracer approach will probably still be the most widely applied technique in the short and intermediate term, due to its high sensitivity and low amounts of tracer which have to be used. Therefore toxicity issues are of less importance compared to MRI or ultrasound imaging probes. In the intermediate to long term, MRI and also ultrasound might be well suited alternative methods, due to their lack of ionizing radiation and high spatial resolution. However, it is likely that not one single parameter, target structure, or imaging technique will be used for assessment of angiogenesis in the future, but a combination of parameters which will allow for evaluation of the angiogenic cascade in its full complexity. Hybrid imaging methods like PET/CT lend themselves for this purpose, as 
they allow for near simultaneous combined molecular and functional imaging, e.g., by combining targeted PET tracers such as $\alpha v \beta 3$-specific compounds and DCE CT. The same potentially applies for future hybrid whole-body MR/PET scanners, which are supposed to be introduced in the near future. Thus a "one-stop-shop" assessment of the different aspects of angiogenesis at the functional and molecular level will hopefully become a reality and might be implemented in therapy planning and response evaluation within the concept of personalized medicine.

\section{Conflicts of interest None.}

\section{References}

1. Folkman J. Tumor angiogenesis: therapeutic implications. N Engl J Med 1971;285:1182-6.

2. Ribatti D, Vacca A, Dammacco F. The role of the vascular phase in solid tumor growth: a historical review. Neoplasia 1999;1:293-302.

3. Risau W. Mechanisms of angiogenesis. Nature 1997;386:671-4.

4. Bergers G, Javaherian K, Lo KM, Folkman J, Hanahan D. Effects of angiogenesis inhibitors on multistage carcinogenesis in mice. Science 1999;284:808-12.

5. Miller JC, Pien HH, Sahani D, Sorensen AG, Thrall JH. Imaging angiogenesis: applications and potential for drug development. J Natl Cancer Inst 2005;97:172-87.

6. Kerbel RS. Antiangiogenic therapy: a universal chemosensitization strategy for cancer? Science 2006;312:1171-5.

7. Hurwitz H, Fehrenbacher L, Novotny W, Cartwright T, Hainsworth J, Heim W, et al. Bevacizumab plus irinotecan, fluorouracil, and leucovorin for metastatic colorectal cancer. N Engl J Med 2004;350:2335-42.

8. Jaffe CC. Measures of response: RECIST, WHO, and new alternatives. J Clin Oncol 2006;24:3245-51.

9. Tortora G, Melisi D, Ciardiello F. Angiogenesis: a target for cancer therapy. Curr Pharm Des 2004;10:11-26.
10. Galbraith SM. Antivascular cancer treatments: imaging biomarkers in pharmaceutical drug development. Br J Radiol 2003;76:S83-6.

11. Kiessling F, Razansky D, Alves F. Anatomical and microstructural imaging of angiogenesis. Eur J Nucl Med Mol Imaging. 2010. doi:10.1007/s00259-010-1450-0.

12. Laking G, Price P. Radionuclide imaging of perfusion and hypoxia. Eur J Nucl Med Mol Imaging. 2010. doi:10.1007/ s00259-010-1453-x.

13. Brix G, Griebel J, Kiessling F, Wenz F. Tracer kinetic modelling of tumour angiogenesis based on dynamic contrast-enhanced CT and MRI measurements. Eur J Nucl Med Mol Imaging. 2010. doi:10.1007/s00259-010-1448-7.

14. Martirosian P, Boss A, Schraml C, Schwenzer NF, Graf H, Claussen $\mathrm{CD}$, et al. Magnetic resonance perfusion imaging without contrast media. Eur J Nucl Med Mol Imaging. 2010. doi:10.1007/s00259-010-1456-7.

15. Cosgrove D, Lassau N. Imaging of perfusion using ultrasound. Eur J Nucl Med Mol Imaging. 2010. doi:10.1007/s00259-010$1537-7$.

16. Haubner R, Beer AJ, Wang H, Chen X. Positron emission tomography tracers for imaging angiogenesis. Eur J Nucl Med Mol Imaging. 2010. doi:10.1007/s00259-010-1503-4.

17. Dijkgraaf I, Boerman OC. Molecular imaging of angiogenesis with SPECT. Eur J Nucl Med Mol Imaging. 2010. doi:10.1007/ s00259-010-1499-9.

18. Lanza GM, Caruthers SD, Winter PM, Hughes MS, Schmieder $\mathrm{AH}, \mathrm{Hu} \mathrm{G}$, et al. Angiogenesis imaging with vascular-constrained particles: the why and how. Eur J Nucl Med Mol Imaging. 2010. doi:10.1007/s00259-010-1502-5.

19. Eisenblätter M, Höltke C, Persigehl T, Bremer C. Optical techniques for the molecular imaging of angiogenesis. Eur $\mathrm{J}$ Nucl Med Mol Imaging. 2010. doi:10.1007/s00259-010-1514-1.

20. Eisenbrey JR, Forsberg F. Contrast-enhanced ultrasound for molecular imaging of angiogenesis. Eur J Nucl Med Mol Imaging. 2010. doi:10.1007/s00259-010-1449-6.

21. Liu Z, Peng R. Inorganic nanomaterials for tumor angiogenesis imaging. Eur J Nucl Med Mol Imaging. 2010. doi:10.1007/ s00259-010-1452-y.

22. Zweifel M, Padhani AR. Perfusion MRI in the early clinical development of antivascular drugs: decorations or decision making tools? Eur J Nucl Med Mol Imaging. 2010. doi:10.1007/ s00259-010-1451-z. 